\title{
Drug Resistance in HIV-1: Genetic and Molecular Bases, Mechanisms and Strategies to Combat the Issue
}

\section{Sharma B*}

Department of Biochemistry, University of Allahabad, India

*Corresponding author: Sharma B, Department of Biochemistry, University of Allahabad, Allahabad, UP, India, Tel: +91-9415715639; E-mail: sharmabi@yahoo.com

Rec date: 06 Oct, 2014; Acc date: 07 Nov, 2014; Pub date: 10 Nov, 2014

Copyright: $\odot 2015$ Sharma B, et al. This is an open-access article distributed under the terms of the Creative Commons Attribution License, which permits unrestricted use, distribution, and reproduction in any medium, provided the original author and source are credited

\section{Editorial}

According to an estimate of WHO, 35.3 million people were living with HIV/AIDS (PLWH) globally at the end of 2012 which included about $0.8 \%$ of adults aged $15-49$ years. According to (NACO) of India, the prevalence of AIDS in India in 2013 was 0.27 million. In low and middle income countries, more than 8 million PLWH are receiving antiretroviral therapy (ART) at the end of 2011. Application of highly active antiretro viral therapy (HAART) worldwide has been able to significantly reduce the death rate of human immunodeficiency virus type 1 (HIV-1) infected individuals. However, the appearance of clinical drug resistance in AIDS patients due to nonadherance to medication (intake of antiretroviral) has been one of the primary reasons associated to chemotherapeutic and virologic failure. In addition, high rate of viral replication, appearance of heterogenous circulating viral quasispecies, infidelity in proviral cDNA synthesis as well as immunological and pharmacological facors are also associated to drug resistance [1]

The virus develops resistance when it evades the effects of the treatment of AIDS patients by antiHIV-1 drugs resulting into no effect on viral replication [2,3]. Antiviral drug resistance is defined by the presence of viral mutations that reduce drug susceptibility compared with the susceptibility of wild-type viruses. It happens because as a retrovirus, HIV employs reverse transcriptase (HIV-1RT) to synthesize a double stranded proviral DNA from its RNA genome. HIV-1RT lacks a proof reading mechanism for correcting errors made during replication of its genome and therefore HIV introduces several mutations in its newly synthesized genomic cDNA [4]. Some of these viral mutants naturally select as drug resistant variants with higher fitness thereby posing serious threat to chemotherapy of AIDS patients $[3,5]$.

WHO has licensed six antiretroviral classes of antiHIV drugs so far which have been found to induce more than 200 mutations in the viral genome. These mutations have been reported to be associated with drug resistance with enhanced fitness: The number of total RT mutations associated with nucleoside/nucleoside reverse transcriptase inhibitor (nRTIs/NRTIs) resistance is more than 50; total number of RT mutations associated with nonnucleoside reverse transcriptase inhibitor (NNRTIs) resistance is more than 40; total mutations associated with protease inhibitor (PIs) resistance are more than 60; total integrase mutations associated with the licensed integrase strand transfer inhibitors are more than 30 and the number of gp41 mutations associated with the fusion / entry inhibitor resistance is more than 15. The mutations in the inhibitor binding sites of coreceptors such as CCR5 or CXCR4 have been reported to cause CCR5 / CXCR4 inhibitor resistance [5,6].

All of these mutations have not been verified via site-directed mutagenesis to confer resistance to a specific drug or drugs. Further, the extent of resistance may be complicated after emergence of any additional mutations in response to application of a specific regimen leading to therapeutic failure [7,8]. A total of 19 antiretroviral drugs approved by WHO for the treatment of HIV-1 infection include 1 nucleotide and 7 nucleoside reverse transcriptase inhibitors (NRTIs), 7 PIs, 3 NNRTIs and 1 fusion/entry inhibitor. The analysis of three dimensional crystal structure of the inhibitor-enzyme complexes and mutational modeling studies have lead to a better understanding of how these drug-resistance mutations exert their effects at a structural level. This information may be exploited towards design and development of new drugs and therapeutic strategies to combat drug resistance to AIDS. A variety of mechanisms have been identified that differ both for different classes of drugs and for drugs of a given class.

The NRTI drugs cause inhibition of the enzyme by competing with the regular dNTPs during DNA synthesis. The type of enzyme inhibition in this case is competitive in nature. The NRTI drug resistance follows two different biochemical mechanisms: (1) mutations mediated resistance that makes HIV-1 RT more fidel so as to discriminate against binding of NRTIs in the catalytic pocket of enzyme during DNA replication, thereby preventing the addition of nucleoside/nucleotide analogs to the growing DNA chain [9-11]. (2) The resistance mediated by those mutations that promote the hydrolytic removal of the chain-terminating NRTI making 3' terminus of the primer containing - $\mathrm{OH}$ group free for continued DNA synthesis $[12,13]$. It occurs via pyrophosphorolysis, nucleotide excision and primer unblocking in association to excess of PPi or ATP in most cells $[14,15]$.

On the other hand, the NNRTIs bind to a hydrophobic pocket in HIV-1 RT located between the $\beta 6-\beta 10-\beta 9$ and $\beta 2-\beta 13-\beta 14$ sheets of the p66 subunit [16] involving a small portion of the pocket from p51 subunit of enzyme. The NNRTIs inhibit HIV-1 replication allosterically by displacing the catalytic aspartate residues relative to the polymerase-binding site $[17,18]$. It results in an altered orientation of the 3' end of the primer terminus thereby not allowing it be accessible to RT to catalyze DNA synthesis. A single mutation in the NNRTI-binding pocket may cause high-level resistance to one or more of the NNRTIs [7]. The mechanism of resistance to NNRTIs may involve the decreased susceptibility of binding of the inhibitor molecules to the enzyme's hydrophobic pocket. The mechanism of actions of fusion inhibitors involves blocking of the conformational change in the gp41 subunit after gp120-CD4-coreceptor binding responsible for fusion of viral and cellular membranes resulting into entry of the viral core into the cell [19]. The viral mutants make the fusion/entry inhibitors ineffective possibly by decrease in binding affinity of inhibitors to gp41. However, in the absence of the drug pressure, the existing canonical transitional mutations such as T215S/C/E/D exhibit ability to convert the mutants into wild type variants turning them to be highly susceptible to the drugs. The 
antagonistic mutations in HIV-1are those mutations which possess ability to generate resistance to one specific drug but they can turn the virus to be more sensitive to a second drug. Very recently, rilpivirine (RPV) and emtricitabine (ETV), the second generation NNRTIs that are known to efficiently inhibit HIV-1 resistant to first generation NNRTIs (nevirapine, delavirdine, and efavirenz), have been found to be ineffective with the appearance of E138K and M184I mutations in RT. The mechanism of viral resistance to RPV has been studied using transient kinetics approaches (quench-flow and stopped-flow) transient kinetics approaches to determine how subunit-specific mutations in p66 or p51 subunits of HIV-1RT influence association and dissociation of RPV to the NNRIs' binding hydrophobic pocket in RT. They have suggested that E138K mutation in p51 confers reduced susceptibility to RPV in presence and absence of M184V mutation [20].

Keeping in view the challenges posed by emergence of potential drug resistant mutants leading to therapeutic failure, serious global efforts are required to be made to design effective strategies to combating this issue in order to eradicate this scourge. The current antiviral research is at the forefront to tackle the drug resistance. According to recent reports, many new antiretroviral targets (both viral and that of host) [21] are being identified and many new structure based small molecules are being developed to potentially block viral replication in infected individuals and also the emergence of drug resistance in them with least or no toxicity [22-24]. Recently researchers have initiated attempting isolation and evaluation of activities of phytochemicals showing aniHIV-1 potential with almost no toxicity to the host [25]. Molecules are needed not only to inhibit the wild type viruses but also to the mutants displaying mono-, di- or multi drug resistance. Recent approaches have focused on development of chimeric molecules (peptide nucleic acid, PNA) involving back bone of repeating $\mathrm{N}$-(2-aminoethyl)-glycine units linked by peptide bonds. The nucleic acid bases (purines and pyrimidines complimentary to the conserved base sequences of LTR, U5PBS,TAR, stem or loop regions of HIV-1 genome) are linked to the backbone by a methylene bridge (-CH2-) and a carbonyl group ($(\mathrm{C}=\mathrm{O})$-) which may specifically mimic viral replication without emergence of drug resistant mutants [26,27]. Apart from suitable retroviral drug development approaches, other strategies such as increasing drug adherence, conducting drug resistance genotyping, the frequent monitoring of patients and drug resistance surveillance, switching the classes of retrovirals in response to resistance and selection of best drugs combination may be employed to combat this issue effectively.

\section{References}

1. Paydary K, Esmaeeli S, Seyed Alinaghi SA, Rouzrokh P, EmamzadehFard S (2013) Emerging HIV Drug Resistance in the Resource-Poor World: Challenges and Strategies. J AIDS Clinic Res S5: 006.

2. Clavel F, Hance AJ (2004) HIV drug resistance. N Engl J Med 350: 1023-1035.

3. Freeman S, Herron JC (2007) Evolutionary Analysis. A case for evolutionary thinking: understanding HIV. (4th edn), Pearson Benjamin Cummings, San Francisco, CA, USA.

4. Roberts JD, Bebenek K, Kunkel TA (1988) The accuracy of reverse transcriptase from HIV-1. Science 242: 1171-1173.

5. Johnson VA, Calvez V, Günthard HF, Paredes R, Pillay D, et al. (2013) Update of the Drug Resistance Mutations in HIV-1: March 2013. Topics in Antiviral Medicine 21: 6-14.
6. Shafer RW, Schapiro JM (2008) HIV-1 drug resistance mutations: an updated framework for the second decade of HAART. AIDS Rev 10: 67-84.

7. Conway B, Wainberg MA, Hall D, Harris M, Reiss P, et al. (2001) Development of drug resistance in patients receiving combinations of zidovudine, didanosine and nevirapine. Aids 15:1269-1274.

8. Ammaranond P, Sanguansittianan S (2012) Mechanism of HIV antiretroviral drugs progress toward drug resistance. Fundam Clin Pharmacol 26: 146-161.

9. Larder BA, Stammers DK (1999) Closing in on HIV drug resistance. Nat Struct Biol 6: 103-106.

10. Larder B (2001) Mechanisms of HIV-1 drug resistance. AIDS 15 Suppl 5: S27-34.

11. Sarafianos SG, Das K, Ding J, Boyer PL, Hughes SH, et al. (1999) Touching the heart of HIV-1 drug resistance: the fingers close down on the dNTP at the polymerase active site. Chem Biol 6: R137-146.

12. Meyer PR, Matsuura SE, Mian AM, So AG, Scott WA (1999) A mechanism of AZT resistance: an increase in nucleotide-dependent primer unblocking by mutant HIV-1 reverse transcriptase. Mol Cell 4: 35-43.

13. Arion D, Sluis-Cremer N, Parniak MA (2000) Mechanism by which phosphonoformic acid resistance mutations restore 3,- azido-3,deoxythymidine (AZT) sensitivity to AZT-resistant HIV-1 reverse transcriptase. J Biol Chem 275:9251-9255.

14. Boyer PL, Sarafianos SG, Arnold E, Hughes SH (2002a) The M184V mutation reduces the selective excision of zidovudine 5 ,-monophosphate (AZTMP) by the reverse transcriptase of human immunodeficiency virus type 1. J Virol 76:3248-3256.

15. Boyer PL, Sarafianos SG, Arnold E, Hughes SH (2002b) Nucleoside analog resistance caused by insertions in the fingers of human immunodeficiency virus type 1 reverse transcriptase involves ATPmediated excision. J Virol 76:9143-9151.

16. Hsiou Y, Ding J, Das K, Clark AD, Boyer PL, et al. (2001) The Lys103Asn mutation of HIV-1 RT: a novel mechanism of drug resistance. J Mol Biol. 309:437-445.

17. Esnouf R, Ren J, Ross C, Jones Y, Stammers D, et al. (1995) Mechanism of inhibition of HIV-1 reverse transcriptase by non-nucleoside inhibitors. Nat Struct Biol 2: 303-308.

18. Spence RA, Kati WM, Anderson KS, Johnson KA (1995) Mechanism of inhibition of HIV-1 reverse transcriptase by nonnucleoside inhibitors. Science 267: 988-993.

19. Eckert DM, Malashkevich VN, Hong LH, Carr PA, Kim PS (1999) Inhibiting HIV-1 entry: discovery of D-peptide inhibitors that target the gp41 coiled-coil pocket. Cell 99: 103-115.

20. Singh K, Marchand B, Rai DK, Sharma B, Michailidis E, et al. (2012) Biochemical mechanism of HIV-1 resistance to rilpivirine. J Biol Chem 287: 38110-38123.

21. Sharma B (2012) Attributes of Host's Genetic Factors in HIV-1 Pathogenesis. Biochemical and Analytical Biochemistry 1:4-7.

22. Sharma B (2011) The antiHIV-1 drugs toxicity and management strategies. Neurobehavioural HIV Medicine, 3: 1-14.

23. Sharma B (2014a) HIV-1, Neuro-AIDS and Cognitive Impairments. J Neuroinfect Dis 5:1-3.

24. Sharma B (2014) Oxidative stress in HIV patients receiving antiretroviral therapy. Curr HIV Res 12: 13-21.

25. Sharma B (2013) Phytochemicals may arrest HIV-1 progression. Clinical Research in HIV AIDS and Prevention 1: 1-5.

26. Nielsen PE, Egholm M (1999) An introduction to peptide nucleic acid. Curr Issues Mol Biol 1: 89-104.

27. Pandey VN, Upadhyay A, Chaubey B (2009) Prospects for antisense peptide nucleic acid (PNA) therapies for HIV. Expert Opin Biol Ther 9: 975-989. 